\title{
EL SURGIMIENTO DE NUEVAS IGLESIAS
}

\author{
Rode, Daniel \\ Seminario Adventista Latinoamericano de Teología \\ isabelydanielrode@gmail.com
}

Fecha de recepción: Noviembre 2013

Fecha de aceptación y versión final: Junio 2014

El presente artículo, muestra que el establecimiento de nuevas iglesias es un quehacer divino, en el que la intervención humana se hace necesaria. Através de experiencias ocurridas en Sudamérica y el mundo sobre el presente tema, ilustra principios importantes en el cumplimiento de la misión cristiana. Enfatiza el hecho de que la razón de ser de la iglesia cristiana es fundar nuevas iglesias, es decir reproducirse para crecer. Finalmente deja abierto el tema ya que el artículo hace parte de una serie de artículos que se iran publicando en los siguientes números de la revista.

Palabras clave: Crecimiento de iglesia, evangelismo, misión. 


\section{THE EMERGENCE OF NEW CHURCHES}

Summary: This article shows that the establishment of new churches is a divine task, in which human intervention is necessary. Through experiences that occurred in South America and the world on this subject, illustrates important principles in the fulfillment of the Christian mission. It emphasizes the fact that the reason for the Christian church is to plant new churches, that is, to reproduce himself for to grow. Finally leaves open the topic, as the article is part of a series of articles that will be published in future issues of the magazine.

Keywords: Church Growth, Evangelism, Mission. 


\section{El interés en el tema}

Establecer nuevas iglesias fue y será la obra de Dios para hacer crecer a su pueblo hasta cubrir la tierra y, esta obra Él siempre quiso hacerla con la colaboración del hombre como su siervo. "Crecimiento de iglesia es aquella disciplina que investiga la naturaleza, expansión, fundación, multiplicación, función y salud de las iglesias cristianas tal como ellas se relacionan con una efectiva implementación de la comisión de Dios de 'Hacer discípulos de todos los pueblos' (Mt 28:19-20)". ${ }^{1}$ El crecimiento de la iglesia moderno también ha incluido la fundación de nuevas congregaciones. Esto no siempre se lo ha tenido en cuenta, pero las iglesias que lo hacen progresan más.

Como profesor de la asignatura Métodos de establecer iglesias en las sedes del Seminario Ad-

1 C. Peter Wagner, Strategies for Church Growth: Tools for Effective Mission and Evangelism (Ventura, California: Regal, 1987), 114; Diccionario hispanoamericano de la mission, "crecimiento de iglesia”. La cursiva fue agregada. ventista Latinoamericano de Teología (de aquí en adelante SALT) de Argentina, Bolivia, Brasil y Perú por más de 19 años, ${ }^{2}$ observo que la Iglesia Adventista del Séptimo Día (de aquí en adelante IASD) ha tomado conciencia de la necesidad de establecer más iglesias. En 2011 las instituciones de la División Sudamericana de la Iglesia Adventista (de aquí en adelante DSA) donaron en torno a tres millones de dólares para la construcción de capillas. En el quinquenio del 2005 a 2010 se establecieron más de 3.000 iglesias y congregaciones esto significó un ritmo del $38 \%$ de Tasa de Crecimiento Decenal (de aquí en adelante TCD). Pero, en el quinquenio de 2010 a 2015 se espera fundar 10.000 iglesias y congregaciones. Se intenta triplicar el "plantío de iglesias". De 22.000 iglesias y congregaciones

2 Comencé a enseñar la asignatura "Métodos de establecer iglesias" en 1994. Ante la solicitud expresa del director del SALT de la DSA, Dr. Wilson Endruveit de enseñar el curso "Evangelización Metropolitana" le sugerí enseñar también "Métodos de establecer iglesias". 
se desea llegar a 32.000, es decir, se quiere tener un ritmo decenal del $112 \%$ TCD. ${ }^{3}$

Ahora es "moda" plantar iglesias $^{4}$ en Latinoamérica. La Unión Uruguaya de la IASD (de aquí en adelante UU) que en los últimos 10 años sólo había establecido tres iglesias; quiere establecer 39 en el quinquenio 2010-2015.5 La Unión Argentina de la IASD (de aquí en adelante UA) intenta organizar más de 300 en el mismo período. En 2011, en las uniones hispanas de la DSA se fundaron 798 iglesias. En la UA se establecieron 122; en la Unión Boliviana, 81; en la Unión Chilena, 84; en la Unión Ecuatoriana, 77; en la Unión Pa-

3 Concilio de pastores de $\mathrm{Foz}$ de Iguazú, 24 al 26 de mayo de 2011; General Conference of Seventh-day Adventists, 147th Annual Statistical Report---2009 (Silver Spring, Maryland: Asociación General de la IASD, 2009), 24; 146th Annual Statistical Report---2008 (Silver Spring, Maryland: Asociación General de la IASD, 2008), 24; 138th Annual Statistical Report---2000 (Silver Spring, Maryland: Asociación General de la IASD, 2000), 26.

4 La expresión "plantar iglesias" es la traducción del inglés "church planting".

5 Encuentro de líderes de la Unión Uruguaya en Blancarena, Uruguay, del 4 al 8 de marzo de 2011. raguaya, 27; en la Unión Peruana del Norte, 123; en la Unión Peruana del Sur, 151; y en la UU, $3 .{ }^{6}$

Las iglesias están tomando conciencia que la misión es primeramente divina aunque con la ayuda de la colaboración humana. Junto con esto, las iglesias están incorporando más formas divinas de hacer la misión. En este primer artículo veremos la toma de conciencia de que la misión es primeramente divina con la colaboración humana. En una segunda parte se verá la toma de conciencia de lo necesario que son las fundaciones de nuevas congregaciones. Finalmente se presentará el plan general de posibles artículos a desarrollar en el futuro.

\section{Toma de conciencia que la misión es misión divina con colaboración humana}

La Iglesia Adventista tiene un mensaje bíblico que compartir con todos los habitantes de

6 "Informe especial, plantando iglesias", Revista Adventista, Febrero 2012, 22-23. 
la tierra, aún con aquellos que pertenecen a otras comunidades cristianas. Pero además, los cristianos creen que "la evangelización mundial requiere que toda la iglesia tome todo el evangelio para todo el mundo". " La organización de nuevas iglesias debe ser entendida como la comisión de Dios que usa el crecimiento de las diversas partes de todas las denominaciones cristianas.

En el Congreso de Lausana en 1974, Ralph Winter ${ }^{8}$ redefinió 'naciones' y 'todas las naciones' (Mt 28:1) como grupos etnolingüísticos, ${ }^{9}$ grupos étnicos o pueblos, no entidades políticas o países. En octubre de 2010 se estimaba que había 17.000 grupos étnicos. En el 41\% de estas etnias, es decir en 7.000 etnias, nunca se había escuchado

7 Norman E. Thomas, "World Mission Conferences: What Impact Do they Have?", International Bolletin of Missionary Research (IBMR) 20, no 4 (octubre 1996): 151. Todas las traducciones del inglés al castellano fueron hechas por el autor. 2009.

8 Ralph Winter falleció en mayo de

9 Allen Yeh, “Tokyo 2010: Global mission Consultation", IBMR 35, $\mathrm{n}^{\circ} 1$ (enero 2011): 5 . del Evangelio. Muchos de estos grupos tienen poca población. ${ }^{10}$ Pero aún así, en 2013, su población era de 2.080.397.000. Es decir, existe un $29,2 \%$ de la población mundial que nunca ha escuchado acerca de Jesús. ${ }^{11}$ Por este motivo, se tomar conciencia de dos cosas: 1) sólo Dios puede lograr la misión mundial y 2) la importancia decisiva de la oración intercesora.

El tema de la Consulta sobre Misión Global de Tokio de mayo de 2010 fue "Hacer discípulos de cada pueblo en nuestra generación”. Esto es un eco del lema de John Mott de 1910, "La evangelización del mundo en esta generación”. Los cristianos con la ayuda de Dios (un enfoque equivocado), en 100 años no pudieron cumplir con ese desafío; y menos se podrá cumplir, con fuerzas humanas, este nuevo

\section{Ibíd., 5-6.}

11 Todd M. Johnson, David Barrett y Peter F. Crossing, "Christianity 2011: Martyrs and the Resurgence of Religions", IBMR, 35, no 1 (2011): 29; "Status of Global Mission, 2013, in the Context of AD 1800-2025", IBMR, 37, n 1, (2013), 33. 
objetivo que es más desafiante: "hacerlos discípulos". 12 En 1950 el cristianismo empezó a despertar a la idea de "Missio Dei" y al uso singular de la "misión".13 No es el hombre con la ayuda de Dios, sino que es la misión de Dios con la colaboración del hombre (1 Co 3:9). Este nuevo paradigma contrastó con la era moderna donde la razón era central. ${ }^{14}$ "Existe la Iglesia porque existe la misión y no al revés". ${ }^{15}$ La misión nace en el corazón de Dios y existe porque Dios ama a las personas. En 1909, Elena de White decía: "Los ángeles del cielo han esperado por mucho tiempo la colaboración de los... miembros de la iglesia en la gran obra que debe hacerse". ${ }^{16}$ En

12 Yeh, “Tokyo 2010”, 6.

13 Walter C. Kaiser, Jr., Mission in the Old Testament: Israel as a Light to the Nations (Grand Rapids, Michigan: Baker Books, 2000), 11.

14 Ahora Dios era central. En la Ilustración, la razón era central. Dios y la iglesia habían sido eliminados (David Bosch, Misión en transformación: cambios de paradigmas en la teología de la misión [Grand Rapids, Michigan: Libros Desafío, 2000], 328).

15 Ibíd., 477.

16 Elena G. de White, Joyas de los junio de 2010; la Conferencia Centenaria Misionera Mundial de Edimburgo vuelve a recordar que "la misión tiene una iglesia". ${ }^{17}$ Esto debiera librar a la iglesia de la soberbia misionera y de una idea estrecha de misión.

David Bosch decía: "La misión es primera y finalmente la obra del Dios trino,...; un ministerio en el cual la Iglesia tiene el privilegio de participar". ${ }^{18}$ No es que Dios tiene una misión para su iglesia en el mundo, sino que Dios tiene una iglesia por causa de su misión en el mundo. "La misión no fue hecha por causa de la iglesia; la iglesia fue hecha por causa de... la misión de Dios". ${ }^{19}$ Entonces, la iglesia de-

Testimonios, 3 vols. (Mountain View, California: Pacific Press, 1953), 3:308-309.

17 Janet Carroll, “Edinburgh 2010 Centennial World Missionary Conference: A Report", IBMR 35, n 1 (2011): 4.

18 Bosch, Misión en transformación, 479.

19 J. Andrew Kirk, What is Mission? Theological Explorations (Londres: Darton, Longman \& Todd, Minneapolis: Fortress Press, 1999), 23-37; Christopher J. H. Wright, The Mission of God: Unlocking the Bible's Grand Narrative (Downers Grove, Illinois: InterVarsity Press, 2006), 62. 
biera cambiar de las concepciones antropocéntricas de misión a una cosmovisión radicalmente teocéntrica. ${ }^{20}$

Del 16 al 25 de octubre de 2010 se celebró en Ciudad del Cabo, Sud África, el tercer Congreso Lausana sobre Evangelización Mundial. Su tema era "Dios en Cristo reconciliando consigo al mundo" (2 Co 5:19). Con 4.000 participantes, este congreso fue considerado el encuentro cristiano global más diverso de los 2.000 años de historia del cristianismo. El

58 desafío fue entender cómo la diversidad cristiana podía colaborar con la misión divina. ${ }^{21} \mathrm{El}$ congreso vibró con el arrepentimiento, la confesión, el deseo de ser creíbles e íntegros en la presentación del Evangelio. En la conclusión del congreso hubo arrepentimiento por el manejo humano de la iglesia, "por la manipulación de los datos, y el uso de las estadísticas para inflar el

20 Wright, The Mission of God, 62.

21 Stanley W. Green, "Report on Cape Town 2010" IBMR, 35, n 1 (enero 2011): 7 . éxito en alcanzar los blancos". ${ }^{22}$ El anhelo era;

Nosotros clamamos a Dios que levante más hombres y mujeres de gracia quienes harán compromisos de largo tiempo para vivir, amar y servir en lugares difíciles dominados por otras religiones, para llevar el perfume y el sabor de Jesucristo entre culturas donde esto es desconocido y es peligroso hacerlo. Esto requiere paciencia y perseverancia, algunas veces por toda una vida, y algunas veces hasta la muerte. ${ }^{23}$

Algo parecido ocurrió en el Concilio Mundial de la IASD, también en octubre de 2010. Los líderes adventistas reconocieron y confesaron humildemente, entre otras cosas, su indiferencia, su mundanalidad, y el manejo humano de sus juntas y prácticas administrativas. ${ }^{24}$ El 11 de

22 Ibíd.

23 Ver sobre el Tercer Congreso Lausana sobre Evangeliación Mundial, del 16-25 de octubre de 2010, en Jonathan J. Bonk, Dwight P. Baker, Craig A. Noll y Rona Johnston Gordon, eds. "The Cape Town Commitment: A Confession of Faith and a Call to Action", IBMR 35, n 2 (Abril de 2011): 72. La cursiva fue agregada.

24 Unión Argentina de la Iglesia 
noviembre de 2010, votaron el documento "Llamado urgente al reavivamiento, la reforma, el discipulado y la evangelización”.25 Los líderes reconocieron su incapacidad para concluir la evangelización mundial y se ponían humildemente al servicio de la misión divina, reconociendo que sólo Dios la puede terminar y lo mínimo que podían hacer es no estorbar dicha misión. En 2009, Raoul Dederen le recordaba a la IASD que,

La iglesia es el cuerpo de Cristo. No se la llamó a la existencia como un fin en sí misma, sino para cumplir el propósito de Dios, es decir, continuar la obra del Señor en el mundo, hacer lo que él haría si todavía estuviera aquí. Esto explica por qué, desde esa perspectiva, la iglesia no tiene simplemente una misión, sino que la iglesia es la misión. ${ }^{26}$

Adventista del Séptimo Día, Llamado urgente al reavivamiento, la reforma, el discipulado y la evangelización (Florida, Buenos Aires: ACES, 2011), 10-11.

25 Ibíd., 7-12.

26 Raoul Dederen, "Capítulo 15: Iglesia”, en Tratado de teología Adventista del Séptimo Día, eds. George W. Reid, Raoul Dederen, Nancy J. Weber de Vyh-
Según lo dicho anteriormente, los diferentes artículos que vendrán invitarán al aprendizaje humilde de iglesias y pastores de diversas denominaciones, y de algunos hechos de la historia de la misión, en especial en Latinoamérica. Se describe como Dios ha usado una variedad de medios para el cumplimiento de su misión. Pero, especialmente, se enfoca en cómo la IASD está tratando de fundar iglesias en Sudamérica colaborando así con la misión divina de llenar la tierra con su gloria. Es decir, se intenta seguir el plan original de Dios de "llenar la tierra" con su gloria mediante: la bendición, "fructificación y multiplicación" del primer hogar-escuela-iglesia (Gn 1:28).

\section{Toma de conciencia de lo necesario que es el estable- cimiento de nuevas iglesias}

$\mathrm{Al}$ vivir en el siglo $\mathrm{XXI}$, con los cambios dramáticos que se producen continuamente, la

meister y Aldo D. Orrego (Florida, Buenos Aires: ACES, 2009), 618. 
iglesia debe abrir sus ojos hacia los campos maduros para la cosecha; y también abrir la mente a nuevas formas de levantar la cosecha. Los cambios tienen mayormente que ver con estructuras funcionales, con renovación de la vida espiritual de las iglesias y el establecimiento de nuevas. Según Malphurs la supervivencia de la Iglesia Evangélica del siglo XXI dependerá de la fundación de iglesias. ${ }^{27}$

Desde su surgimiento en 1990, el programa Misión Global impulsó la fundación de iglemundial tuvo un $77 \%$ TCD en su feligresía. De 6.183.585 miembros, en 1989, pasó a 10.939.182, en 1999. Y tuvo un $67 \%$ TCD de crecimiento en sus iglesias y congregaciones, de 56.095 en 1989 pasó a 93.648 en 1999. Aunque este crecimiento en iglesias es loable, aún se estaba 10 puntos o $13 \%{ }^{28}$ por

27 Aubrey Malphurs, Planting Growing Churches for the 21st Century (Grand Rapids, Michigan: Baker, 1992), 32-45.

28 Entre $77 \%$ y $67 \%$ hay 10 puntos de diferencia. Estos 10 puntos divididos 77

debajo del crecimiento en miembros. ${ }^{29} \mathrm{El}$ problema fue que en la última década de 2001 a 2011 la tasa de crecimiento en miembros bajó al $42 \%$ de TCD y el crecimiento de iglesias y congregaciones bajó al $30 \%$ de TCD. ${ }^{30}$ De esta forma la diferencia entre la TCD en miembros y la TCD en nuevas iglesias se distanció más, la diferencia fue de 12 puntos, equivalente al $30 \%$ menos. $^{31}$ De todas maneras, para fin de 2012 se tenían unas 145.000 iglesias y congregaciones. ${ }^{32}$ En

y multiplicados por 100 nos dan $13 \%$.

29 Global Mission, "A 10-Year Report", Adventist News Network (Silver Spring, Maryland, USA), disponible en www.globalmission.org; Internet (consultada del 10 de diciembre de 2003).

30 De 12.320.844 miembros en 2001 se pasó a 17.479 .890 en 2011; y de 107.153 iglesias y congregaciones se pasó a 139.223 (General Conference of Seventh-day Adventists, 139th Annual Statistical Report-2011 [Silver Spring, Maryland: General Conference of Seventh-day Adventists, 2001], 36; 149th Annual Statistical Report-2011, 42).

31 Entre 49 y 41 hay 8 puntos. Estos 8 puntos divididos por 49 y multiplicados por 100 da $16 \%$.

32 En 2009 había 132.025 iglesias y congregaciones que crecían a un $41 \%$ TCD equivalente al 3,50\% TCA. El estimado 
el siglo XXI, pareciera comenzar un despertar respecto a la responsabilidad de fundar iglesias. En 2002, George Knight decía:

En la Iglesia Adventista están sucediendo cosas emocionantes. La siembra de iglesias está cobrando nueva vida, hecho especialmente importante en lugares... donde se había perdido el interés. Pero ahora, centenares...de nuevas congregaciones se están estableciendo cada año. ... El objetivo es capturar nuevo territorio para Cristo. ${ }^{33}$

Primeramente, para seguir progresando, la iglesia tendrá que transformar las iglesias existentes, lo que no es tan fácil, se dice que es "más fácil tener un bebé que levantar un muerto". Además, como segundo paso, tendrá que abrir nuevas iglesias con una mentalidad más agresiva focalizándose especialmente en las migraciones a las grandes urbes. Es bueno recordar que: "El método de evangelizar

para 2012 da unas 145.000 iglesias y congregaciones.

33 George R. Knight, Por la ruta de Romanos (Buenos Aires: ACES, 2002), 352. más efectivo que hay debajo del cielo es el de establecer iglesias nuevas". ${ }^{34}$ Hace unos 40 años, Wagner descubrió que en el mismo lugar donde algunas iglesias estaban declinando otras iglesias estaban creciendo. Las que crecían eran las que enfatizaban entre otras cosas el establecimiento de nuevas congregaciones. ${ }^{35}$ Desde entonces, este mismo hecho ha sido comprobado por diversos estudios en distintas denominaciones en todo el mundo.

Las mayores satisfacciones personales las he tenido en la apertura de nuevas congregaciones. La apostasía de los recién bautizados llena de sombras los recuerdos hermosos que tienen los pastores. Pero da satisfacción, observar la integración a la misión de las iglesias que se ayudó a fundar. ${ }^{36}$ En mi trabajo

34 C. Peter Wagner, Plantando iglesias para una mayor cosecha (Miami, Florida: Unilit, 1997), 11.

35 C. Peter Wagner, Your Church can be Healthy (New York, NY: Harper \& Row, 1979), 24-28.

36 Esta satisfacción el autor la tuvo en: Las Piedras, Montevideo; Las Heras, Mendoza; San Luis; Río Cuarto, Córdoba; La Picada, Paraná Este, Paraná Sur, Barrio 
como ministro participé en la apertura de diferentes iglesias. Me tocó colaborar como anciano y pastor, evangelista, administrador de campo y profesor. Esto me permitió vivenciar los pormenores del antes, durante y después del establecimiento de una nueva congregación. Estos artículos son producto de esas vivencias personales.

Los reportes de cualquier denominación que informan sobre el crecimiento de miembros también adicionan el crecimiento de nuevas iglesias y todo reporte de decrecimiento en miembros implica el decrecimiento del número de iglesias. ${ }^{37}$ Esto también se notó en la IASD en la última década, ${ }^{38}$ en los lugares donde se ha continuado plantando iglesias, la feligresía creció.

Gasano, Ramírez, Gualeguay, Comunidad Hebrea de Libertador San Martín, Entre Ríos; Rafaela, Sunchales, Galvez, Santa Fe; Río Tercero, Córdoba; Iglesia Juvenil de San Fernando, Estados Unidos; y la Comunidad Hebrea de Buenos Aires, entre otras.

37 División Norteamericana de la Iglesia Adventista del Séptimo Día, The Church Planter, edición Especial (Boise, Idaho: Pacific Press, 1997), 11, 13.

38Ver el comienzo de esta introducción.
Por ejemplo, la Iglesia Adventista en Israel se triplicó entre 1995 a 2000, de 400 miembros pasó a 1200. Tuvo un $800 \%$ de TCD, esto significó diez veces más que el crecimiento de la IASD en el ámbito mundial en el mismo quinquenio. El presidente de la Iglesia Adventista en Israel, explica que este crecimiento se debió al énfasis otorgado a la creación de nuevas congregaciones y a la relevancia del adventismo en las diversas extracciones culturales. En los últimos cinco años la IASD en Israel pasó de 5 a 25 iglesias y congregaciones. ${ }^{39}$ Es decir, las comunidades religiosas adventistas se quintuplicaron, tuvieron un $400 \%$ de TCD.

\section{Plan general de los artículos}

Los artículos tienen dos grandes partes. La primera parte consta de seis artículos que encuentran en la historia y la Biblia el fundamento de por qué

39 "Hechos y realizaciones", Revista Adventista, noviembre 2002, 16. 
y cómo establecer iglesias. La segunda parte se desarrolla en cuatro artículos que sistematizan las maneras más comunes de cómo han sido fundadas y, también, podrían ser fundadas las iglesias.

En la parte I ya vimos el interés en el tema. El siguiente artículo tratará de presentar algunas de las principales razones bíblicas y prácticas de por qué los fundadores de iglesias consideran sumamente importantes establecer nuevas iglesias. Otro artículo desarrollará algunos ejemplos de cómo se iniciaron iglesias cristianas de diversas denominaciones en el marco de la realidad Latinoamericana. Los dos artículos siguientes presentarán la información de cómo surgían las comunidades religiosas pequeñas en el Antiguo Testamento, en el Nuevo Testamento y en los comienzos de la Iglesia Adventista. Se cerrará esta parte I tratando las dificultades más comunes en el establecimiento de una nueva iglesia.

La parte II se iniciará con un artículo mostrando el fenómeno bíblico, actual y práctico de una de las mejores y más efectivas formas de fundar iglesias urbanas en nuevos municipios, esto es, por medio de equipos de jóvenes. Por supuesto, esto no implica menospreciar lugares geográficos o personas de otras edades que están abriendo iglesias de diferentes maneras y en diversos lugares. Los dos artículos siguientes tratarán veinte formas distintas de iniciar iglesias. Primero se verán nueve maneras de abrir una nueva congregación a partir de una iglesia madre. Luego se presentarán once formas de hacerlo a partir de agencias misioneras o asociaciones de iglesias. El siguiente artículo comentará la manera de potenciar todas las maneras de establecer iglesias, se trata de las nuevas tecnologías de comunicación. Estas diversas formas de establecer iglesias sólo se presentan a fin de sistematizar el estudio. En la práctica las formas de hacerlo son infinitas. La mayoría de las veces, en el inicio de una iglesia se combinan varias maneras, y generalmente tienen como eje una o dos de ellas como centro. 
En el artículo de cierre, se hará una conclusión que tratará de enlazar todas las ideas con el pensamiento principal del tema, esta misión divina que comienza en el Edén y que podrá ser concluida en la medida que los seres humanos sean siervos de Dios. Esta serie de artículos se basan en el AT, en el NT y en las raíces de la IASD. Pero también se apoyan en las formas que se abrieron históricamente las iglesias cristianas en Sudamérica; en reconocimientos in situ de diversas iglesias prestigiosas; en entrevistas a experimentados misioneros fundadores de iglesias especialmente de Sudamérica; $\mathrm{y}$ en investigaciones y estudios de casos realizados por el autor y los estudiantes de teología de posgrado de casi todos los países de Latinoamérica, la mayoría de los cuales son pastores de amplia experiencia en fundar iglesias en diversos contextos de Latinoamérica. ${ }^{40}$ Estos aportes pragmá-

40 El autor ha tenido como sus discípulos a estudiantes de Maestría y Doctorado que habían fundado entre 10 a 60 iglesias en diversos contextos de los diferentes países de Latinoamérica. ticos de varios años conforman un marco para este estudio que le otorga la seriedad de una investigación erudita y práctica al mismo tiempo.

El siguiente artículo tratará sobre las razones principales para establecer iglesias que proponen ciertos fundadores de diferentes denominaciones cristianas. Pero, la razón fundamental para hacerlo es seguir el plan original de Dios de cubrir geográficamente la tierra con su gloria por medio de la multiplicación de hogares-escuelas-iglesias parecidas a la primera comunidad religiosa familiar establecida en el Edén. 